\title{
Phospho-ERK and AKT status, but not KRAS mutation status, are associated with outcomes in rectal cancer treated with chemoradiotherapy
}

\author{
Janine M Davies ${ }^{1,2^{*}}$, Dimitri Trembath ${ }^{3}$, Allison M Deal ${ }^{2,4}$, William K Funkhouser ${ }^{3}$, Benjamin F Calvo ${ }^{2,5}$, \\ Timothy Finnegan ${ }^{6}$, Karen E Weck ${ }^{3}$, Joel E Tepper ${ }^{2,7}$ and Bert $\mathrm{H}^{\mathrm{O}}$ Neil $^{1,2}$
}

\begin{abstract}
Background: KRAS mutations may predict poor response to radiotherapy. Downstream events from KRAS, such as activation of BRAF, AKT and ERK, may also confer prognostic information but have not been tested in rectal cancer (RC). Our objective was to explore the relationships of KRAS and BRAF mutation status with p-AKT and p-ERK and outcomes in RC.

Methods: Pre-radiotherapy RC tumor biopsies were evaluated. KRAS and BRAF mutations were assessed by pyrosequencing; $\mathrm{p}-\mathrm{AKT}$ and $\mathrm{p}$-ERK expression by immunohistochemistry.

Results: Of 70 patients, mean age was 58; 36\% stage II, 56\% stage III, and 9\% stage IV. Responses to neoadjuvant chemoradiotherapy: 64\% limited, 19\% major, and 17\% pathologic complete response. 64\% were KRAS WT, 95\% were BRAF WT. High p-ERK levels were associated with improved OS but not for p-AKT. High levels of $\mathrm{p}$-AKT and p-ERK expression were associated with better responses. KRAS WT correlated with lower p-AKT expression but not p-ERK expression. No differences in OS, residual disease, or tumor downstaging were detected by KRAS status.

Conclusions: KRAS mutation was not associated with lesser response to chemoradiotherapy or worse OS. High p-ERK expression was associated with better OS and response. Higher p-AKT expression was correlated with better response but not OS.
\end{abstract}

Keywords: Rectal cancer, KRAS analysis, phosphoERK, phosphoAKT, radiation response

\section{Background}

The most common genetic mutation in all cancers is the RAS mutation, occurring in $30 \%$ of cancers [1]. The RAS family of genes $(\mathrm{K}-, \mathrm{H}-$, and $\mathrm{N}-\mathrm{RAS})$ transduce, and likely integrate, messages from growth factor receptors [2]. Intracellular pathway signaling via the $R A S$ pathway causes cell proliferation and invasion along with evasion of apoptosis [2,3]. In rectal cancer $(\mathrm{RC})$ series that included patients with stage IV disease, KRAS mutations, are reported in 19 to $48 \%$ of patients[4-6]. This is similar to metastatic colorectal cancer ( $\mathrm{mCRC}$ ) in which mutations are found in 30 to $40 \%$ of patients, mostly at codons

\footnotetext{
* Correspondence: janine.davies@bccancer.bc.ca

'Department of Medicine, Division of Hematology/Oncology, University of North Carolina at Chapel Hill, 170 Manning Dr, CB 7305, Chapel Hill, NC 27599-7305, USA

Full list of author information is available at the end of the article
}

12, 13, and 61. Mutation of these codons of KRAS leads to constitutive activation of the RAS signaling pathway. There are conflicting data with regards to the predictive and prognostic significance of KRAS mutations in colon cancer, and less is known for RC. However, in an initial assessment of RAS oncogene expression in $\mathrm{RC}$, one retrospective study evaluated the RAS gene protein product, p21 [7]. Higher p 21 titers were associated with worse 5 year survival ( 44 vs. $64 \%, \mathrm{p}<0.02$ ), more frequent distant metastases ( 52 vs. $23 \%, \mathrm{p}<0.001$ ), and more advanced stage ( 54 vs. $36 \%$ incidence of Dukes' C, $\mathrm{p}<0.04$ ) [7].

$R A F$ is one of several targets of activated $R A S$, and itself is mutated in approximately 8 to $10 \%$ of $\mathrm{mCRC},[8]$ and 0 to $12 \%$ specifically in $\mathrm{RC}[5,6,9,10]$. There is strong evidence to suggest that $K R A S$ and $B R A F$ mutations are mutually exclusive $[8,11-13]$. Constitutive activation of $B R A F$ leads to extracellular signal-regulated kinase (ERK) 
phosphorylation (p-ERK) and activation [14]. ERK is required for cell proliferation and allows for the evasion of apoptosis [14]. To date, only small studies have been reported in $\mathrm{mCRC}$, but these suggest that outcomes are worse among patients with $B R A F$ mutations $[8,13]$. In $\mathrm{RC}$, this has not been corroborated and the effect(s) of ERK has not been elucidated.

The phosphatidylinositol 3-kinase (PI3K)/AKT pathway is another downstream activation target of mutated $K R A S$, and is important in cell metabolism, growth, motility, survival, proliferation, and metastasis [15-18]. Following activation through receptor kinases or activated RAS, PI3K phosphorylates and activates AKT (pAKT) [19]. Activation of AKT by PI3K is inhibited by the tumor suppressor gene PTEN,[19] which is occasionally lost via mutation or gene silencing in CRC $[20,21]$. In small cell lung cancer, elevated phosphorylated AKT was associated with limited stage disease but was not prognostic [22]. In prostate cancer, AKT expression intensity has been associated with serum levels of prostate specific antigen [23]. In breast cancer, p-AKT expression was inversely correlated with survival [24]. Ionizing and UV radiation are known to activate the PI3K/AKT pathway [25]. Activating mutations of the PI3K p110 subunit are found in 10 to $30 \%$ of colon cancers $[17,26,27]$. In $\mathrm{mCRC}$, it is not clear if mutation of PI3K or activation of the pathway has an impact on survival or other outcomes $[19,26]$.

Based on the above preclinical and clinical findings, the objective of this study was to explore the relationships of $K R A S$ and BRAF status with p-AKT and p-ERK expression and with clinical outcomes (response to radiation and overall survival $[\mathrm{OS}]$ ) in rectal adenocarcinoma. Many downstream targets of RAS can be inhibited by small molecule agents, yet potential consequences of such inhibition are not known. The principal rationale was to determine which RAS-activated pathways are relevant to radiation (RT) resistance in order to select appropriate targets for therapy. Both KRAS and BRAF mutations were hypothesized to confer radioresistance and result in lesser response to RT and worse survival. Activation of either AKT or ERK was also hypothesized to antagonize chemoradiotherapy effects, be associated with worse survival, and be associated with mutations of KRAS or BRAF.

\section{Methods}

This was a retrospective study of RC patients identified through the Institutional Tumor Registry, who received preoperative chemoradiotherapy with concurrent $5 \mathrm{FU}$ chemotherapy followed by surgical resection, and had adequate tissue for evaluation in the preoperative biopsy and/or surgical resection sample. Clinical data were collected from the patients' medical records with complete follow-up until April 23, 2008, at which point survival data were censored. Conduct of the study was performed with approval by our Institutional Review Board and in accordance with the Helsinki Declaration of 1975, as revised in 2000.

The primary clinical outcome measure for this study was pathologic response, which was divided into three objective categories: limited response (pLR, gross residual disease present), major response (pMR, only microscopically visible disease remaining), or complete response ( $\mathrm{pCR}$, no pathologic evidence of residual tumor cells). AJCC pathologic stage at surgery was also assessed and compared with preoperative staging to determine treatment response (downstaging).

\section{Mutation analysis}

$K R A S$ and BRAF mutation testing were performed by pyrosequencing as described elsewhere,[28-31] using preoperative or postoperative samples. All samples were enriched for tumor cells by microdissection prior to mutation testing. An H\&E stained slide prepared from formalin-fixed paraffin embedded tissue samples was examined under a light microscope by a pathologist and an area containing at least $50 \%$ tumor cells was microdissected from adjacent unstained slides for tumor enrichment prior to xylene deparaffinization and DNA extraction. DNA was extracted using the Qiagen DNeasy Tissue Extraction Kit from tissue samples pretreated with xylene for removal of paraffin. Pyrosequencing was performed to identify KRAS mutations in codons 12, 13 and 61 and the BRAF V600E mutation using the PyroMark Q96 KRAS v2.0 kit (\#972452, Qiagen) and PyroMark $B R A F$ RUO Kit (\#40-0057, Qiagen), respectively, as per the manufacturer instructions. PCR reactions were performed on a Veriti thermal cycler (Applied Biosystems) and pyrosequencing was performed on the PyroMark MD (Pyrosequencing AB). A positive control, normal control, and blank (no DNA template) PCR control were included in each assay. Pyrograms were analyzed by PyroMark 1.0 software using allele quantification (AQ) mode to determine the percentage of mutant versus wild-type alleles according to relative peak height.

\section{Immunohistochemistry (IHC)}

was performed on preoperative biopsies using previously published methods [32]. Briefly, unstained 5-micron-thick sections were baked at $60^{\circ} \mathrm{C}$ for 15 to 60 minutes. Baked sections were soaked twice in fresh xylene for 5 minutes each, then soaked in 100\% ethanol for 3 minutes, and blocked for endogenous peroxidase with 3\% hydrogen peroxide in methanol for 10 minutes. Slides were soaked in 95\% ethanol for 3 minutes, $70 \%$ ethanol for 3 minutes, rinsed in distilled water and soaked in Dako wash buffer (Dako Cat. No. S3006; Dako, Glostrup, Denmark) for 5 minutes. Slides were then steamed in a Black \& Decker 
steamer for 25 minutes using antigen retrieval buffers (Dako) for each primary antibody to be studied (Ser-473 antibody for p-AKT, Cell Signaling Systems, Cat. No. 736E11; Thr-202/Tyr-204 antibody for p-ERK, Cell Signaling Systems, Ca. No. D13.14.4E) and then allowed to cool for at least 20 minutes. Sections were transferred to Dako wash buffer for 5 minutes. Endogenous biotin was neutralized by incubating the slides in a biotin blocking system (Dako Cat. No. X0590) for 10 minutes at room temperature in each of the 2 solutions. Sections were then exposed to the primary antibodies (Ser-473 antibody at 1:25 dilution; Thr-202/Tyr-204 antibody at 1:200 dilution) for 30 minutes at room temperature. After rinsing in Dako wash buffer, slides were incubated with the Dako LSAB2 biotinylated link for 10 minutes at room temperature, rinsed in Dako wash buffer, and then incubated with the Dako LSAB2 streptavidin-horseradish peroxidase for 10 minutes at room temperature. After additional rinsing in Dako wash buffer, detection of the antibody/antigen complex was visualized using 3-3 diaminobenzidine for 5 minutes. Slides were then rinsed in water, lightly counterstained in filtered Mayer's hematoxylin, rinsed, dehydrated, cleared, and mounted. The cells of interest (tumor cells) in each section were scored for percentage reactivity and signal strength in both the cytoplasm and the nuclei. Simultaneously stained normal rectal mucosa and no primary antibody stained normal mucosa served as negative controls in each experiment.

Nuclear peroxidase staining (chosen because it was more consistent from sample to sample than cytoplasmic staining) was scored by one surgical pathologist (WKF) who was blinded to clinical information. Sections were scored by multiplying the average staining intensity seen on a scale of 0 to $3+$ by the percentage of cells that were positive to any degree, creating a range of possible scores of 0 to 300 .

\section{Statistical Methods}

Categorical data were analyzed using Fisher's Exact Test. Continuous data comparing pathological response and stage with p-AKT and p-ERK activation used JonckheereTerpstra tests to account for ordered differences among groups, and Wilcoxon Rank Sum tests were used to compare p-AKT and p-ERK among mutation groups. Survival curves were created using the Kaplan Meier method and were compared using the Log rank test; OS was calculated from the time of radiation treatment. Cox regression analyses were used to explore the association of p-AKT and p-ERK activation with OS. Analyses were performed using SAS v9.2 statistical software.

\section{Results}

Patient characteristics are summarized in Table $1(\mathrm{n}=70)$. Six patients $(9 \%)$ had stage IV disease at diagnosis for
Table 1 Patient characteristics and clinical data

\begin{tabular}{llc}
\hline Characteristics & & $\mathbf{N}=\mathbf{7 0}$ \\
\hline Age at diagnosis & Median & 58 years \\
Gender & Range & $26-89$ \\
& Male & $42(60 \%)$ \\
Race & Female & $28(40 \%)$ \\
& White & $52(74 \%)$ \\
& Black & $15(21 \%)$ \\
Clinical disease stage (at diagnosis) & Other or unknown & $3(4 \%)$ \\
& III & $25(36 \%)$ \\
Pathological disease response & IV & $39(56 \%)$ \\
& Complete response (pCR) & $12(17 \%)$ \\
& Major response (pMR) & $13(19 \%)$ \\
Treatment response & Limited response (pLR) & $45(64 \%)$ \\
& Downstaged & $30(43 \%)$ \\
& No change & $33(47 \%)$ \\
Recurrence & Upstaged & $7(10 \%)$ \\
& No & $45(64 \%)$ \\
Status (censored April 23, 2008) & Alive & $25(36 \%)$ \\
& Dead & $7(10 \%)$ \\
& Yes & $13(19 \%)$ \\
& Local recurrence & $5(7 \%)$ \\
& Distant recurrence & $41(59 \%)$ \\
& Both local and distant & $29(41 \%)$ \\
& &
\end{tabular}

pCR: Complete response; pMR: Major response; pLR: Limited response.

which initial treatment with chemoradiotherapy was selected. At the time of surgery, $43 \%$ of patients were downstaged and $17 \%$ had a pCR. All but two patients received concurrent 5-FU chemotherapy with the radiation. There was no association of age, sex, nor race with stage at diagnosis (all $\mathrm{p}>0.2$ ).

With a median follow-up for survivors of 42 months, $36 \%$ experienced recurrence, $72 \%$ of which were distant recurrences. For all patients, the median OS was 4.5 years (95\% CI 3.0-12.5).

KRAS and BRAF mutation testing was successfully performed on 67 and 64 of the tumors, respectively. Among evaluable samples, 24 (36\%) were mutant for KRAS and 3 (5\%) were mutant for BRAF (Table 2). $K R A S$ and $B R A F$ mutations were mutually exclusive except in one patient (who had a pCR and therefore further tissue was not available) in which the tumor was positive for both KRAS and BRAF mutations.

\section{KRAS/BRAF mutations do not predict chemoradiation response in rectal cancer}

One of our principal hypotheses based on preclinical information was that KRAS mutation would confer radioresistance and result in reduced response to $\mathrm{RT}$. Our results, however, did not confirm this hypothesis (Table 2). There was no difference in residual tumor 
Table 2 KRAS and BRAF mutational status with outcomes

\begin{tabular}{|c|c|c|}
\hline & KRAS $(n=67)$ & $B R A F(n=64)$ \\
\hline Wild-type (WT) & $43(64 \%)$ & 61 (95\%) \\
\hline Mutant & 24 (36\%) & $3(5 \%)$ \\
\hline Median OS WT & 4.1 years & 4.1 years \\
\hline Median OS mutant & 4.9 years $p=0.6$ & Not reached $p=0.1$ \\
\hline \multicolumn{3}{|l|}{ WT vs. mutant } \\
\hline Limited response & $67 \%$ vs. $67 \%$ & $69 \%$ vs. $33 \%$ (1 of 3 ) \\
\hline Major response & $14 \%$ vs. $21 \%$ & $16 \%$ vs. $0 \%$ \\
\hline Complete response & $19 \%$ vs. $13 \% p=0.7$ & $15 \%$ vs. $66 \%$ (2 of 3 ) \\
\hline \multicolumn{3}{|l|}{ WT vs. mutant } \\
\hline Downstaged & $42 \%$ vs. $38 \%$ & $36 \%$ vs. $66 \%$ (2 of 3 ) \\
\hline $\begin{array}{l}\text { No change or } \\
\text { upstaged }\end{array}$ & $58 \%$ vs. $63 \% p=0.8$ & $64 \%$ vs. $33 \%$ (1 of 3 ) \\
\hline \multicolumn{3}{|l|}{ Recurrence } \\
\hline None & $67 \%$ vs. $54 \%$ & $62 \%$ vs. $100 \%$ (3 of 3 ) \\
\hline Local recurrence & $12 \%$ vs. $8 \%$ & $10 \%$ \\
\hline Distant recurrence & $14 \%$ vs. $29 \%$ & $20 \%$ \\
\hline $\begin{array}{l}\text { Both local and } \\
\text { distant }\end{array}$ & $7 \%$ vs. $8 \% p=0.5$ & $8 \%$ \\
\hline
\end{tabular}

WT: Wildtype; OS: Overall Survival.

status, difference in radiation response as assessed by downstaging of tumors, or frequency of local recurrence based on KRAS or BRAF status.

\section{KRAS/BRAF mutations do not predict overall survival in rectal cancer}

There was no difference in OS by KRAS mutation status (median OS 4.1 years for WT vs. 4.9 years for mutated tumors, $\mathrm{p}=0.6$, Table 2 ), consistent with recent results in $\mathrm{mCRC}$ [33]. The number of patients with $B R A F$ mutation was small and we did not find a significant difference in OS by BRAF mutation status (median OS 4.1 years for WT vs. not reached for mutated tumors, $\mathrm{p}=$ $0.1)$. Interestingly, none of the three $B R A F$ mutant patients died during follow-up, a finding that is limited given the small number of patients.

\section{AKT activation status, but not ERK activation status, correlates with KRAS mutation status}

Because ERK and AKT are both potential targets of activated/mutated $K R A S$, we explored correlations between KRAS mutation and phosphorylation status of these molecules. Representative examples of low and high staining intensity are demonstrated for pAKT and pERK (Figure 1). Median nuclear p-AKT staining intensity was 80 (range 0-300). Patients with mutant KRAS tumors had higher p-AKT scores $($ mean $=109)$ than $K R A S \mathrm{WT}$ tumors (mean $=67, \mathrm{p}=0.04$, Figure 2A). Median nuclear p-ERK staining intensity was 80 (range 0-300). Surprisingly, and in contrast to $\mathrm{p}-\mathrm{AKT}$, there was no difference noted in $\mathrm{p}$-ERK intensity by KRAS mutation status ( $\mathrm{p}=$ 0.5 , Figure $2 \mathrm{~B})$. No significant association was noted between p-ERK levels and $B R A F$ mutational status in this small group of patients.

\section{p-AKT and p-ERK activation are associated with better response to chemoradiotherapy}

Based on existing preclinical information, we hypothesized that activation of either AKT or ERK would antagonize chemoradiotherapy effect based on inhibition of apoptosis $[34,35]$. To test this, we examined associations between $\mathrm{p}$-AKT or p-ERK activation status defined by IHC with chemoradiotherapy response and survival. Our results stand in contradiction to our hypothesis, however, in that increasing intensity of both $\mathrm{p}$-AKT and p-ERK were associated with less residual disease suggestive of better responses to RT (Figure 3). Patients with pCR or pMR at the time of surgery had higher p-AKT levels than those with $\mathrm{pLR}(\mathrm{p}=0.006)$. When the three pathologic response categories were compared by p-ERK levels, differences were noted ( $p=0.02)$. $p$-ERK levels were significantly higher in the pMR group, but not in the pCR group, when compared to the pLR ( $p=0.03,0.07$ respectively).

\section{p-ERK activation is associated with survival, but not p- AKT}

We lastly hypothesized that either p-ERK or p-AKT activation would be associated with worse survival. On the contrary, patients with tumor p-ERK expression above the median had significantly longer survival compared with those below ( 12.5 vs. 3.0 years, $p=0.016$, Figure $4 B$ ). This survival difference was maintained but lost statistical significance when the stage IV patients were excluded from the analysis $(p=0.06)$. Overall, for each 50 point decrease in $\mathrm{p}$-ERK staining intensity, the risk of death increased by $32 \%(\mathrm{p}=0.06)$, reflecting a benefit to increased $\mathrm{p}$-ERK levels. A borderline significant improvement in OS was also detected with lesser staining intensity of $\mathrm{p}-\mathrm{AKT}$ ( $\mathrm{p}=$ 0.08 , Figure $4 \mathrm{~A})$. When the stage IV patients were excluded, patients with tumor p-AKT expression below the median had significantly longer survival than those below the median $(\mathrm{p}=0.029)$.

\section{Discussion}

In $\mathrm{RC}$ patients treated with chemoradiotherapy, KRAS mutation was not associated with lesser response to chemoradiotherapy or worse OS. While both p-AKT and pERK expression were correlated with better response to chemoradiotherapy, only high p-ERK expression was associated with better OS.

Constitutive activation of $K R A S$ via mutation is a common finding in CRC, and one that has been assumed to be of importance to tumorigenesis due to its frequent and early occurrence in the adenoma-carcinoma sequence. Recent studies in $\mathrm{mCRC}$ have demonstrated 


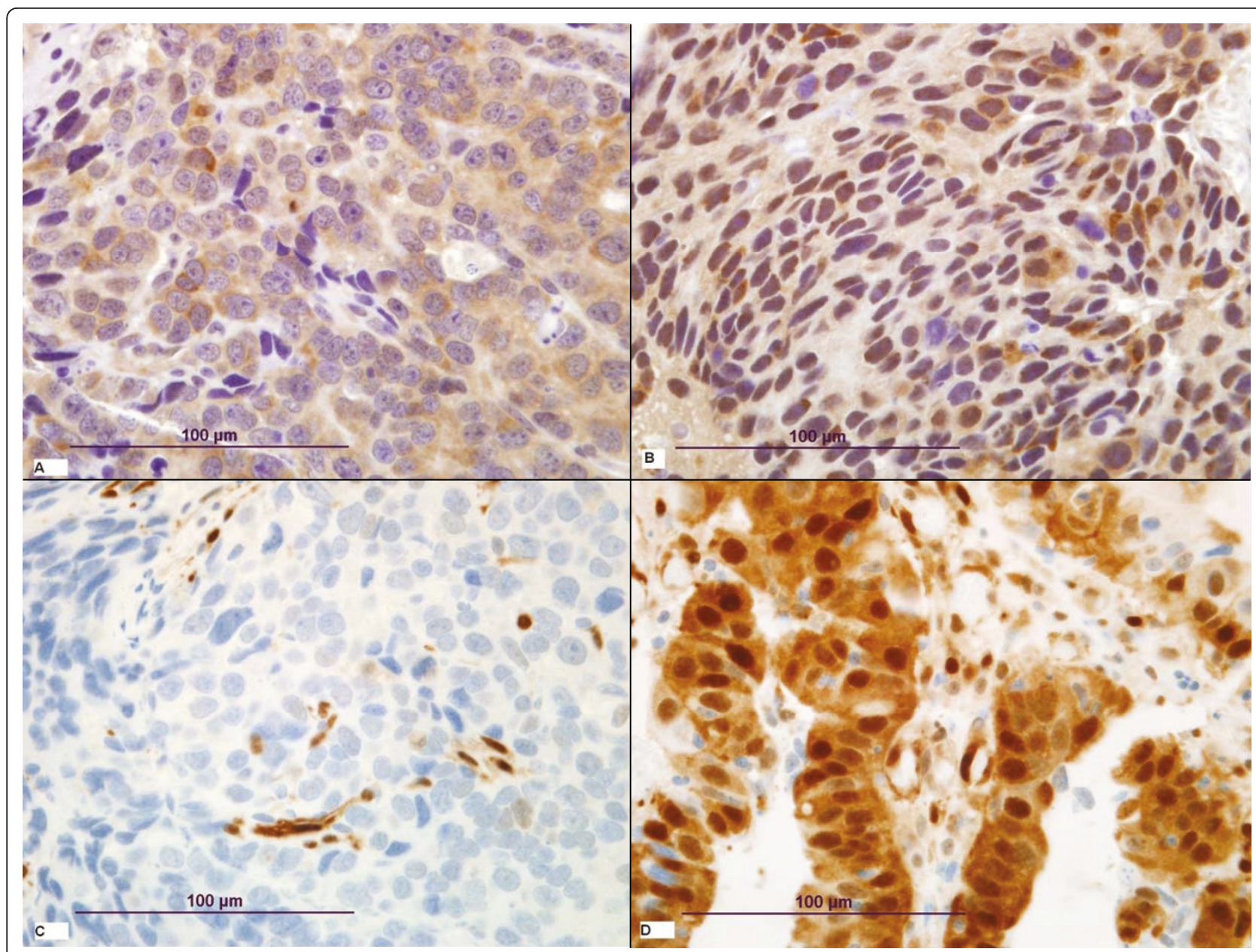

Figure 1 Representative examples of immunohistochemistry staining intensity for p-AKT (low intensity, panel A; high intensity, panel B) and p-ERK (low intensity, panel C; high intensity, panel D) at $60 \times$ magnification.

that responses to epidermal growth factor receptor (EGFR) inhibitors (cetuximab or panitumumab) occur only in patients with the wild-type (WT) KRAS gene. These drugs are now used in the treatment of mCRC, with response rates ranging from $8 \%$ with single agent cetuximab[36] to $23 \%$ in combination with irinotecan, [37] and disease control rates (a combination of complete and partial responses and stable disease) of 39 and $56 \%$ respectively.

Preclinical studies have suggested that RAS mutation would lead to radioresistance, and conversely that targeting RAS or downstream effectors of activated KRAS would identify potential tumor-specific radiosensitizers [38-40]. This in part formed the rationale for targeting of EGFR in RC, an intervention that has to date been disappointing in clinical trials. Studies of RAS in human $\mathrm{RC}$ tumor samples have generally been small and have produced mixed conclusions [2,5,7,41-43]. These studies have reported no change in downstaging[41] or disease free status by KRAS status [5]. Other studies found that
KRAS WT was associated with improved survival,[2] or trended to tumor downstaging[7] or response,[42] while another study found associations between KRAS mutation with earlier stage and better survival [43]. Our study set out to confirm that KRAS mutational status was associated with radiosensitivity using more modern sequencing technology in a larger number of patients than had been studied previously. Our findings did not confirm KRAS mutation as a prognostic factor, nor as a predictive factor for resistance to chemoradiotherapy, suggesting that $K R A S$ may not be a worthwhile target to pursue as a radiosensitizer in $\mathrm{RC}$.

Our findings confirm a relatively low rate of $B R A F$ mutation as is seen in mCRC, with numbers too small to draw conclusions about the effect of BRAF on chemoradiotherapy response. However, we note that all three patients with confirmed $B R A F$ mutations were long-term survivors.

This study also represented an opportunity to assess the effects of KRAS mutation on activation of its downstream 

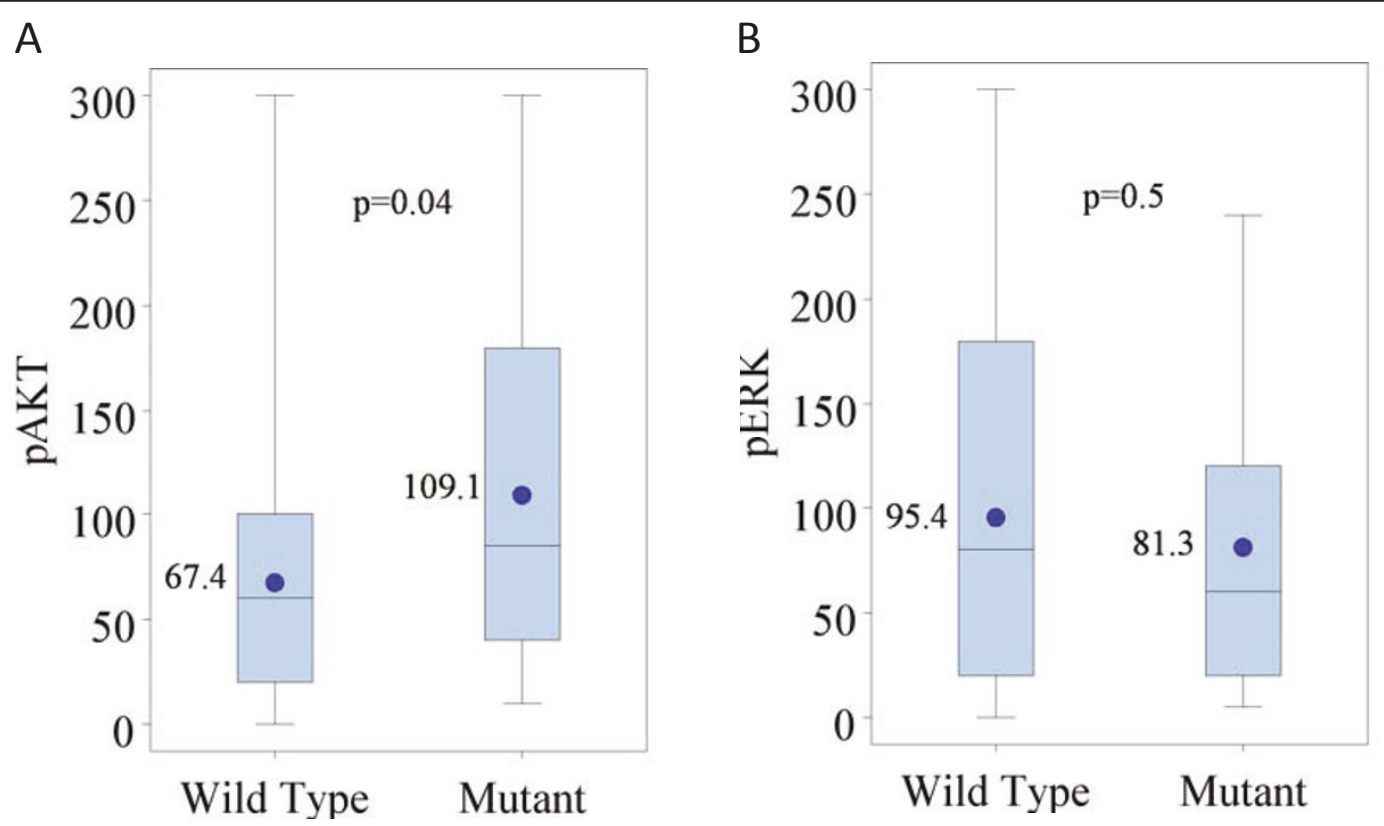

Figure 2 p-AKT (A; wildtype $n=41$, mutant $n=24$ ) and $p$-ERK (B; wildtype $n=42$, mutant $n=23$ ) expression by KRAS mutational status.

targets ERK and AKT. Mutation or activation of KRAS results in constitutive activation of several downstream effectors, and to date it has not been clear which effectors are most important in mediating the effects of mutated $K R A S$ in cancer cells [1]. We found that AKT activation was significantly associated with $K R A S$ activation, while ERK activation was not. One potential weakness of this finding, however, is that anti-phosphoprotein antibodies were used in paraffin-embedded tissues of various ages with the possibility that there was variability in the stability of the phosphorylated proteins. Another small study of predominantly stage II and III CRC tumors evaluated the $K R A S / B R A F / E R K$ pathway. p-ERK was correlated with $K R A S$ codon 12 mutations $(\mathrm{p}=0.016)$ but not with codon 13 mutations [14]. BRAF mutations (V599) were infrequent $(8.7 \%)$ and not associated with p-ERK status [14]. We did not examine the association between mutation location and p-ERK in our study. Preoperative sample limitations prevented all phosphorylation and mutational analyses to be conducted on these untreated samples.

Among our most interesting findings was that higher p-ERK levels were associated with better survival and
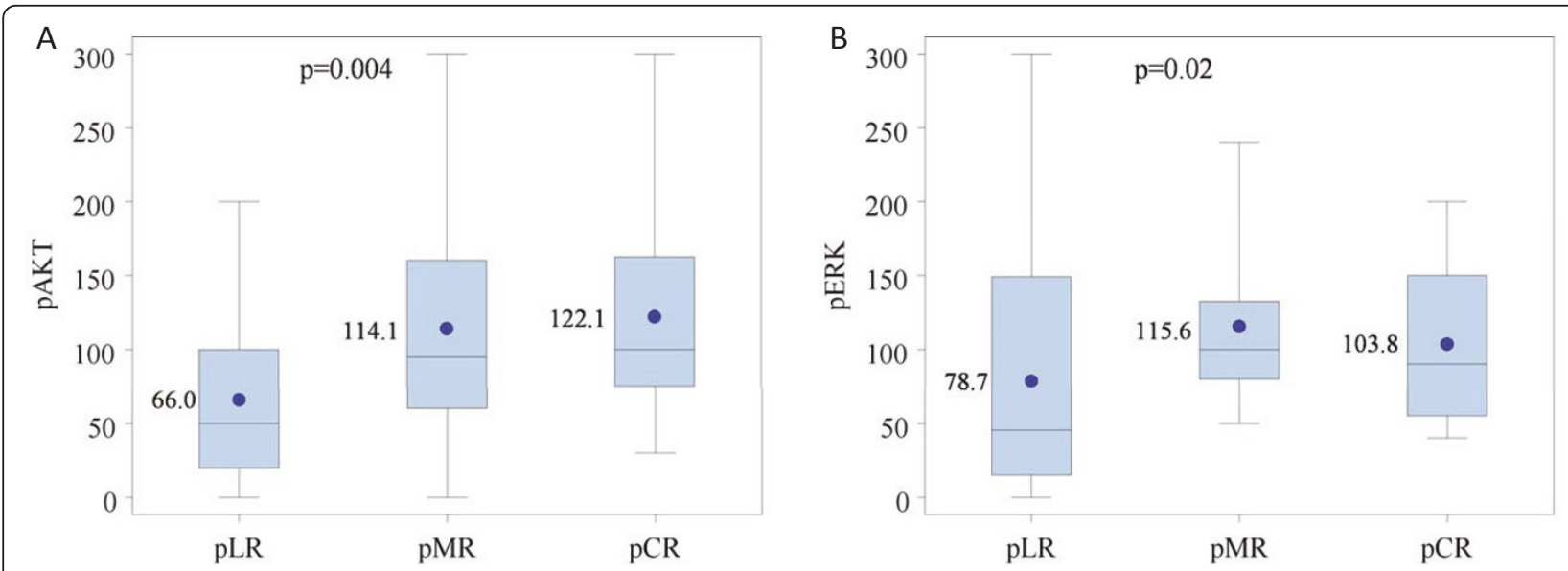

Figure 3 p-AKT ( $A$; pLR $n=43$, pMR $n=13, p C R n=12$ ) and $p$-ERK ( $B$; $p L R n=44$, pMR $n=12, p C R n=12$ ) expression by radiation response (residual disease at the time of surgery) with responses categorized as limited response (pLR), major response (pMR), or complete response $(\mathrm{pCR})$. 

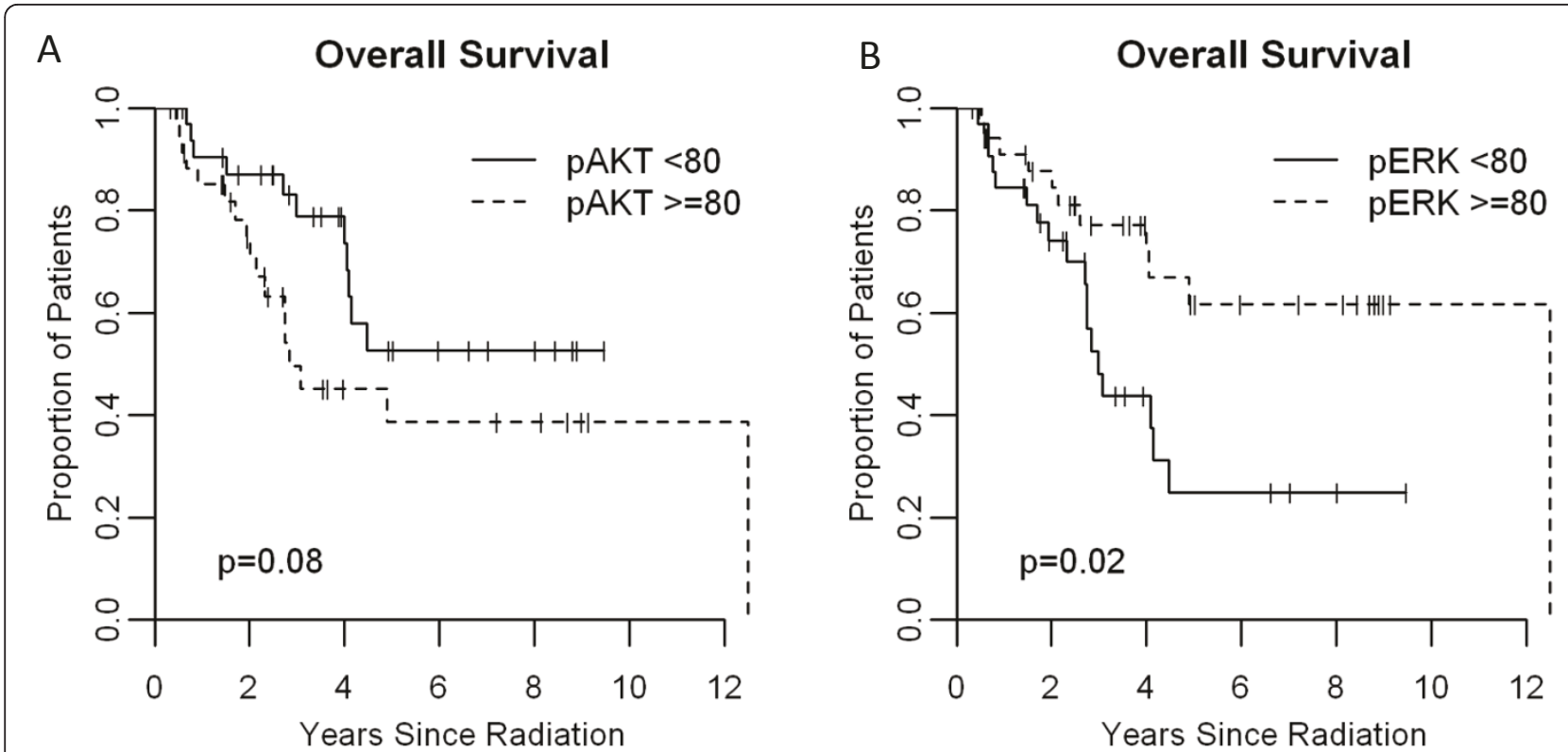

Figure 4 Overall survival by p-AKT ( $A$; $p$-AKT $<80, n=33, p$-AKT $\geq 80, n=35$ ) and p-ERK (B; $p$-ERK $<80, n=32, p$-ERK $\geq 80 n=36$ ) expression.

response to chemoradiotherapy, and that higher $\mathrm{p}$-AKT expression was also associated with better response to chemoradiotherapy. Certain tumors exhibiting activated $\mathrm{AKT}$, including squamous cell cancer of the head and neck[44] and cervical cancer,[34] have been shown to respond poorly to chemoradiotherapy. The positive association between ERK activation and response is less surprising, as ERK activation results in increased cell cycling,[45] and presence of higher fractions of cycling cells has previously been associated with better outcome from chemoradiotherapy for RC [46]. The positive association between ERK activation and survival is the first described in RC to our knowledge. In hepatocellular carcinoma, higher pERK staining intensity was associated with longer time to progression [47].

It is more difficult to understand why AKT activation would be beneficial in terms of response. Data from other tumor types suggests that activation of the AKT cell survival pathway confers resistance to radiation $[34,44]$. For example, in a PTEN-deficient glioma model, a case in which AKT is constitutively active, PTEN gene transfer resulted in significant radiosensitization [48]. In a recent publication, it was demonstrated that PI3K mutations, which would be expected to result in constitutive AKT activation, were associated with a higher rate of local recurrence of RC (27.8 vs. 9.4\%) [6].

\section{Conclusions}

In summary, we assessed RAS and selected downstream effectors in order to determine the relationships between KRAS (and BRAF) with these effectors and clinical outcomes. Our results suggest that activation of AKT and ERK may be beneficial for response to radiation therapy, and thus targeting these pathways in the setting of chemoradiotherapy could possibly be contraindicated and should be evaluated prospectively with a sufficient sample size.

\section{List of Abbreviations}

ERK: extracellular signal-regulated kinase; IHC: immunohistochemistry; mCRC: metastatic colorectal cancer; OS: overall survival; p-AKT phosphorylated AKT; pCR: pathologic complete response; p-ERK: phosphorylated extracellular signal-regulated kinase; PI3K: phosphatidylinositol 3-kinase (AKT); pLR: pathologic limited response; pMR: pathologic major response; RC: rectal cancer; RT: radiation

\section{Acknowledgements}

We appreciate the assistance of Kay C. Chao for performing the KRAS testing, Chris Civalier for performing the BRAF testing, Nana Feinberg for performing the IHC testing, and the Anatomic Pathology Core Laboratory. This work was supported by the GI SPORE (P50 CA 106991), National Institutes of Health (K23 CA118431-04 to BHO), Alberta Heritage Foundation for Medical Research Clinical Fellowship (to JMD), and Canadian Association of Medical Oncology Fellowship (to JMD).

This study was presented at the $2010 \mathrm{Gl}$ Cancers Symposium, Orlando FL, and the 2010 Canadian Association of Medical Oncologists Annual Scientific Meeting, Montreal, QC.

\section{Author details}

${ }^{1}$ Department of Medicine, Division of Hematology/Oncology, University of North Carolina at Chapel Hill, 170 Manning Dr, CB 7305, Chapel Hill, NC 27599-7305, USA. ${ }^{2}$ UNC Lineberger Comprehensive Cancer Center, University of North Carolina at Chapel Hill, School of Medicine, CB 7295, Chapel Hill, NC, 27599-7295, USA. ${ }^{3}$ Department of Pathology and Laboratory Medicine, University of North Carolina at Chapel Hill, Brinkhous-Bullitt Building, CB 7525, Chapel Hill, NC, 27599-7525, USA. ${ }^{4}$ Lineberger Biostatistics Core, UNC Lineberger Comprehensive Cancer Center, University of North Carolina at 
Chapel Hill, School of Medicine, CB 7295, Chapel Hill, NC, 27599-7295, USA. ${ }^{5}$ Department of Surgery, Division of Surgical Oncology, University of North Carolina at Chapel Hill, 170 Manning Dr, CB 7213, Chapel Hill, NC 275997213, USA. ${ }^{6}$ Alamance Regional Medical Center, 1240 Huffman Mill Rd, Burlington, NC, 27215, USA. 'Department of Radiation Oncology, University of North Carolina at Chapel Hill, 170 Manning Dr, CB 7305, Chapel Hill, NC 27599-7305, USA.

\section{Authors' contributions}

$\mathrm{BHO}$ conceived the study and design, coordinated the study, prepared IHC samples, participated in the data analysis, interpretation of results, and drafted the manuscript. KW and DT carried out the molecular genetic studies. KW participated in the data interpretation. WKF carried out the immunohistochemical studies. TF participated in clinical data collection. AMD performed the statistical analyses. JMD participated in the data analyses, interpretation of results, and drafted the manuscript. BFC participated in the study development and data analysis. JET participated in the study design, data interpretation and manuscript revisions. All authors read and approved the final manuscript.

\section{Competing interests}

The authors declare that they have no competing interests.

Received: 20 April 2011 Accepted: 12 September 2011

Published: 12 September 2011

\section{References}

1. Cox AD, Der CJ: Ras family signaling: therapeutic targeting. Cancer Biol Ther 2002, 1:599-606

2. Jimeno A, Messersmith WA, Hirsch FR, Franklin WA, Eckhardt SG: KRAS mutations and sensitivity to epidermal growth factor receptor inhibitors in colorectal cancer: practical application of patient selection. J Clin Oncol 2009, 27:1130-1136.

3. Castagnola P, Giaretti W: Mutant KRAS, chromosomal instability and prognosis in colorectal cancer. Biochim Biophys Acta 2005, 1756:115-125.

4. Bengala C, Bettelli S, Bertolini F, Sartori G, Fontana A, Malavasi N, Depenni R, Zironi S, Del Giovane C, Luppi G, Conte PF: Prognostic role of EGFR gene copy number and KRAS mutation in patients with locally advanced rectal cancer treated with preoperative chemoradiotherapy. Br J Cancer 2010, 103:1019-1024.

5. Gaedcke J, Grade M, Jung K, Schirmer M, Jo P, Obermeyer C, Wolff HA, Herrmann MK, Beissbarth T, Becker $\mathrm{H}$, et al: KRAS and BRAF mutations in patients with rectal cancer treated with preoperative chemoradiotherapy. Radiother Oncol 2010, 94:76-81.

6. He Y, Van't Veer L, Mikolajewska-Hanclich I, van Velthuysen ML, Zeestraten EC, Nagtegaal ID, van de Velde CJ, Marijnen CA: PIK3CA mutations predict local recurrences in rectal cancer patients. Clin Cancer Res 2009, 15:6956-6962.

7. Michelassi F, Vannucci LE, Montag A, Chappell R, Rodgers J, Block GE: Ras oncogene expression as a prognostic indicator in rectal adenocarcinoma. J Surg Res 1988, 45:15-20.

8. Di Nicolantonio F, Martini M, Molinari F, Sartore-Bianchi A, Arena S, Saletti P, De Dosso S, Mazzucchelli L, Frattini M, Siena S, Bardelli A: Wild-type BRAF is required for response to panitumumab or cetuximab in metastatic colorectal cancer. J Clin Oncol 2008, 26:5705-5712.

9. Fransen $K$, Klintenas M, Osterstrom A, Dimberg J, Monstein HJ, Soderkvist P: Mutation analysis of the BRAF, ARAF and RAF-1 genes in human colorectal adenocarcinomas. Carcinogenesis 2004, 25:527-533.

10. Kalady MF, Sanchez JA, Manilich E, Hammel J, Casey G, Church JM: Divergent oncogenic changes influence survival differences between colon and rectal adenocarcinomas. Dis Colon Rectum 2009, 52:1039-1045.

11. Deng G, Bell I, Crawley S, Gum J, Terdiman JP, Allen BA, Truta B, Sleisenger MH, Kim YS: BRAF mutation is frequently present in sporadic colorectal cancer with methylated $\mathrm{hMLH} 1$, but not in hereditary nonpolyposis colorectal cancer. Clin Cancer Res 2004, 10:191-195.

12. Frattini M, Balestra D, Suardi S, Oggionni M, Alberici P, Radice P, Costa A, Daidone $M G$, Leo E, Pilotti $S$, et al: Different genetic features associated with colon and rectal carcinogenesis. Clin Cancer Res 2004, 10:4015-4021.

13. Tol J, Nagtegaal ID, Punt CJ: BRAF mutation in metastatic colorectal cancer. N Engl J Med 2009, 361:98-99.
14. Georgieva M, Krasteva M, Angelova E, Ralchev K, Dimitrov V, Bozhimirov S, Georgieva E, Berger MR: Analysis of the K-ras/B-raf/Erk signal cascade, p53 and CMAP as markers for tumor progression in colorectal cancer patients. Oncol Rep 2008, 20:3-11.

15. Engelman JA, Luo J, Cantley LC: The evolution of phosphatidylinositol 3kinases as regulators of growth and metabolism. Nat Rev Genet 2006, 7:606-619.

16. Manning BD, Cantley LC: AKT/PKB signaling: navigating downstream. Cell 2007, 129:1261-1274

17. Samuels Y, Wang Z, Bardelli A, Silliman N, Ptak J, Szabo S, Yan H, Gazdar A, Powell SM, Riggins GJ, et al: High frequency of mutations of the PIK3CA gene in human cancers. Science 2004, 304:554.

18. Courtney KD, Corcoran RB, Engelman JA: The PI3K pathway as drug target in human cancer. J Clin Oncol 2010, 28:1075-1083.

19. Ogino S, Nosho K, Kirkner GJ, Shima K, Irahara N, Kure S, Chan AT, Engelman JA, Kraft P, Cantley LC, et al: PIK3CA mutation is associated with poor prognosis among patients with curatively resected colon cancer. J Clin Oncol 2009, 27:1477-1484.

20. Nassif NT, Lobo GP, Wu X, Henderson CJ, Morrison CD, Eng C, Jalaludin B, Segelov E: PTEN mutations are common in sporadic microsatellite stable colorectal cancer. Oncogene 2004, 23:617-628.

21. Goel A, Arnold CN, Niedzwiecki D, Carethers JM, Dowell JM, Wasserman L, Compton C, Mayer RJ, Bertagnolli MM, Boland CR: Frequent inactivation of PTEN by promoter hypermethylation in microsatellite instability-high sporadic colorectal cancers. Cancer Res 2004, 64:3014-3021.

22. Blackhall FH, Pintilie M, Michael M, Leighl N, Feld R, Tsao MS, Shepherd FA: Expression and prognostic significance of kit, protein kinase $B$, and mitogen-activated protein kinase in patients with small cell lung cancer. Clin Cancer Res 2003, 9:2241-2247.

23. Liao Y, Grobholz R, Abel U, Trojan L, Michel MS, Angel P, Mayer D: Increase of AKT/PKB expression correlates with gleason pattern in human prostate cancer. Int J Cancer 2003, 107:676-680.

24. Schmitz KJ, Otterbach F, Callies R, Levkau B, Holscher M, Hoffmann O, Grabellus F, Kimmig R, Schmid KW, Baba HA: Prognostic relevance of activated Akt kinase in node-negative breast cancer: a clinicopathological study of 99 cases. Mod Pathol 2004, 17:15-21.

25. Zhan M, Han ZC: Phosphatidylinositide 3-kinase/AKT in radiation responses. Histol Histopathol 2004, 19:915-923.

26. Kato S, lida S, Higuchi T, Ishikawa T, Takagi Y, Yasuno M, Enomoto M, Uetake H, Sugihara K: PIK3CA mutation is predictive of poor survival in patients with colorectal cancer. Int J Cancer 2007, 121:1771-1778.

27. Samuels Y, Velculescu VE: Oncogenic mutations of PIK3CA in human cancers. Cell Cycle 2004, 3:1221-1224.

28. Weichert W, Schewe C, Lehmann A, Sers C, Denkert C, Budczies J, Stenzinger $\mathrm{A}$, Joos $\mathrm{H}$, Landt $\mathrm{O}$, Heiser $\mathrm{V}$, et al: KRAS genotyping of paraffinembedded colorectal cancer tissue in routine diagnostics: comparison of methods and impact of histology. J Mol Diagn 2010, 12:35-42.

29. Spittle C, Ward MR, Nathanson KL, Gimotty PA, Rappaport E, Brose MS, Medina A, Letrero R, Herlyn M, Edwards RH: Application of a BRAF pyrosequencing assay for mutation detection and copy number analysis in malignant melanoma. J Mol Diagn 2007, 9:464-471.

30. Tan YH, Liu Y, Eu KW, Ang PW, Li WQ, Salto-Tellez M, lacopetta B, Soong R: Detection of BRAF V600E mutation by pyrosequencing. Pathology 2008, 40:295-298.

31. Tsiatis AC, Norris-Kirby A, Rich RG, Hafez MJ, Gocke CD, Eshleman JR, Murphy KM: Comparison of Sanger Sequencing, Pyrosequencing, and Melting Curve Analysis for the Detection of KRAS Mutations. Diagnostic and Clinical Implications. J Mol Diagn 2010.

32. Puvvada SD, Funkhouser WK, Greene K, Deal A, Chu H, Baldwin AS, Tepper JE, O'Neil BH: NF-kB and Bcl-3 Activation Are Prognostic in Metastatic Colorectal Cancer. Oncology 2010, 78:181-188.

33. Van Cutsem E, Kohne CH, Hitre E, Zaluski J, Chang Chien CR, Makhson A, D'Haens G, Pinter T, Lim R, Bodoky G, et al: Cetuximab and chemotherapy as initial treatment for metastatic colorectal cancer. N Engl J Med 2009, 360:1408-1417.

34. Kim TJ, Lee JW, Song SY, Choi JJ, Choi CH, Kim BG, Lee JH, Bae DS: Increased expression of PAKT is associated with radiation resistance in cervical cancer. Br J Cancer 2006, 94:1678-1682.

35. Bonni A, Brunet A, West AE, Datta SR, Takasu MA, Greenberg ME: Cell survival promoted by the Ras-MAPK signaling pathway by transcriptiondependent and -independent mechanisms. Science 1999, 286:1358-1362. 
36. Jonker DJ, O'Callaghan CJ, Karapetis CS, Zalcberg JR, Tu D, Au HJ, Berry SR, Krahn M, Price T, Simes RJ, et al: Cetuximab for the treatment of colorectal cancer. N Engl J Med 2007, 357:2040-2048.

37. Cunningham D, Humblet $Y$, Siena $S$, Khayat D, Bleiberg H, Santoro A, Bets D, Mueser M, Harstrick A, Verslype C, et al: Cetuximab monotherapy and cetuximab plus irinotecan in irinotecan-refractory metastatic colorectal cancer. N Engl J Med 2004, 351:337-345.

38. McKenna WG, Weiss MC, Bakanauskas VJ, Sandler H, Kelsten ML, Biaglow J, Tuttle SW, Endlich B, Ling CC, Muschel RJ: The role of the H-ras oncogene in radiation resistance and metastasis. Int I Radiat Oncol Biol Phys 1990, 18:849-859.

39. Jones HA, Hahn SM, Bernhard E, McKenna WG: Ras inhibitors and radiation therapy. Semin Radiat Oncol 2001, 11:328-337.

40. Bernhard EJ, Stanbridge EJ, Gupta S, Gupta AK, Soto D, Bakanauskas VJ, Cerniglia GJ, Muschel RJ, McKenna WG: Direct evidence for the contribution of activated $\mathrm{N}$-ras and $\mathrm{K}$-ras oncogenes to increased intrinsic radiation resistance in human tumor cell lines. Cancer Res 2000 60:6597-6600.

41. Grann A, Zauber P: Is there a predictive value for molecular markers in predicting response to radiation and chemotherapy in rectal cancer? Int J Radiat Oncol Biol Phys 2002, 54:1286-1287.

42. Bengala C, Bettelli S, Bertolini F, Salvi S, Chiara S, Sonaglio C, Losi L, Bigiani N, Sartori G, Dealis C, et al: Epidermal growth factor receptor gene copy number, K-ras mutation and pathological response to preoperative cetuximab, 5-FU and radiation therapy in locally advanced rectal cancer. Ann Oncol 2009, 20:469-474.

43. Luna-Perez P, Segura J, Alvarado I, Labastida S, Santiago-Payan H, Quintero A: Specific c-K-ras gene mutations as a tumor-response marker in locally advanced rectal cancer treated with preoperative chemoradiotherapy. Ann Surg Oncol 2000, 7:727-731.

44. Gupta AK, MCKenna WG, Weber CN, Feldman MD, Goldsmith JD, Mick R, Machtay M, Rosenthal DI, Bakanauskas VJ, Cerniglia GJ, et al: Local recurrence in head and neck cancer: relationship to radiation resistance and signal transduction. Clin Cancer Res 2002, 8:885-892.

45. Seger R, Krebs EG: The MAPK signaling cascade. FASEB J 1995, 9:726-735.

46. Willett CG, Warland G, Hagan MP, Daly WJ, Coen J, Shellito PC, Compton CC: Tumor proliferation in rectal cancer following preoperative irradiation. J Clin Oncol 1995, 13:1417-1424.

47. Abou-Alfa GK, Schwartz L, Ricci S, Amadori D, Santoro A, Figer A, De Greve J, Douillard J-Y, Lathia C, Schwartz B, et al: Phase II Study of Sorafenib in Patients With Advanced Hepatocellular Carcinoma. J Clin Oncol 2006, 24:4293-4300.

48. Wick W, Furnari FB, Naumann U, Cavenee WK, Weller M: PTEN gene transfer in human malignant glioma: sensitization to irradiation and CD95L-induced apoptosis. Oncogene 1999, 18:3936-3943.

doi:10.1186/1748-717X-6-114

Cite this article as: Davies et al:: Phospho-ERK and AKT status, but not KRAS mutation status, are associated with outcomes in rectal cancer treated with chemoradiotherapy. Radiation Oncology 2011 6:114.

\section{Submit your next manuscript to BioMed Central and take full advantage of:}

- Convenient online submission

- Thorough peer review

- No space constraints or color figure charges

- Immediate publication on acceptance

- Inclusion in PubMed, CAS, Scopus and Google Scholar

- Research which is freely available for redistribution

Submit your manuscript at www.biomedcentral.com/submit
Biomed Central 